\title{
RANDOMIZED PICK-FREEZE FOR SPARSE SOBOL INDICES ESTIMATION IN HIGH DIMENSION
}

\author{
Yohann de CAStro $^{1}$ And Alexandre Janon ${ }^{1}$
}

\begin{abstract}
This article investigates selection of variables in high-dimension from a non-parametric regression model. In many concrete situations, we are concerned with estimating a non-parametric regression function $f$ that may depend on a large number $p$ of inputs variables. Unlike standard procedures, we do not assume that $f$ belongs to a class of regular functions (Hölder, Sobolev, ... ), yet we assume that $f$ is a square-integrable function with respect to a known product measure. Furthermore, observe that, in some situations, only a small number $s$ of the coordinates actually affects $f$ in an additive manner. In this context, we prove that, with only $\mathcal{O}(s \log p)$ random evaluations of $f$, one can find which are the relevant input variables with overwhelming probability. Our proposed method is an unconstrained $\ell_{1}$-minimization procedure based on the Sobol's method. One step of this procedure relies on support recovery using $\ell_{1}$-minimization and thresholding. More precisely, we use a thresholdedLASSO to faithfully uncover the significant input variables. In this frame, we prove that one can relax the mutual incoherence property (known to require $\mathcal{O}\left(s^{2} \log p\right)$ observations) and still ensure faithful recovery from $\mathcal{O}\left(s^{\alpha} \log p\right)$ observations for any $1 \leq \alpha \leq 2$.
\end{abstract}

Mathematics Subject Classification. 62G08, 62G35, 65H10, 93A30, $93 \mathrm{~B} 35$.

Received September 21, 2014. Revised March 30, 2015.

\section{INTRODUCTION}

\subsection{Sensitivity analysis and Sobol indices}

Some mathematical models encountered in applied sciences involve a large number of poorly-known parameters as inputs. It is important for the practitioner to assess the impact of this uncertainty on the model output. An aspect of this assessment is sensitivity analysis, which aims to identify the most sensitive parameters, that is, parameters having the largest influence on the output. The parameters identified as influent have to be carefully tuned (or estimated) by the users of the model. On the other hand, parameters whose uncertainty has a small impact can be set to a nominal value (which can be some special value, for which the model is simpler).

In global (stochastic) variance-based sensitivity analysis (see for example [22] and references therein), the input variables are assumed to be independent random variables. Their probability distributions account for the practitioner's belief about the input uncertainty. This turns the model output into a random variable, whose total variance can be split down into different partial variances (this is the so-called Hoeffding decomposition,

Keywords and phrases. Sensitivity analysis, Sobol indices, high-dimensional statistics, LASSO, Monte-Carlo method.

1 Université Paris-Sud, Laboratoire de Mathématiques d’Orsay, Bâtiment 425, Université Paris-Sud, 91405 Orsay, France.

alexandre.janon@math.u-psud.fr 
also known as functional ANOVA, see [18]). Each of these partial variances measures the uncertainty on the output induced by each input variable uncertainty. By considering the ratio of each partial variance to the total variance, we obtain a measure of importance for each input variable that is called the Sobol's index or sensitivity index of the variable [23,24]; the most sensitive parameters can then be identified and ranked as the parameters with the largest Sobol indices. Each partial variance can be written as the variance of the conditional expectation of the output with respect to each input variable.

Once the Sobol indices have been defined, the question of their effective computation or estimation remains open. In practice, one has to estimate (in a statistical sense) those indices using a finite sample (of size typically in the order of hundreds of thousands) of evaluations of model outputs [27]. Indeed, many Monte Carlo or quasi Monte Carlo approaches have been developed by the experimental sciences and engineering communities. This includes the Sobol pick-freeze (SPF) scheme (see [13,24]). In SPF a Sobol index is viewed as the regression coefficient between the output of the model and its pick-freezed replication. This replication is obtained by holding the value of the variable of interest (frozen variable) and by sampling the other variables (picked variables). The sampled replications are then combined to produce an estimator of the Sobol index.

\subsection{High-dimensional contexts}

The pick-freeze scheme is used on models with a reasonable (typically, less than one thousand) number of inputs. When there is a large number of input parameters (what we call a high-dimensional context), this scheme will require a number of model evaluations which is generally too large to be computed in practice. Hence, in high-dimensional contexts, some specific sensitivity analysis methods exist, such as screening methods (for instance, Morris's scheme [21]), but they do not target the estimation of Sobol indices. Note that in [28], an interesting method for estimating Sobol indices is proposed and seems to be applicable in high-dimensional contexts. This method is not based on a sparsity assumption ( $c f$. infra) like ours, and is not applied on models with more than one hundred parameters. Moreover, it is worth noting that alternative Monte-Carlo pick-freeze schemes can be preferable for estimating small Sobol indices [25]; however this does not adress the curse of dimensionality, in the sense that the required number of evaluations of $f$ is still proportional to the total number of input variables.

Besides, models with a large number of input parameters often display a so-called sparsity of effects property, that is, only a small number of input parameters are actually influent: in other terms, we want to efficiently estimate a sparse vector of Sobol indices. Sparse estimation in high-dimensional contexts is the object of highdimensional statistics methods, such as the LASSO estimator.

In our frame, we would like to find the most influent inputs of a unknown function that is to be described. This framework is closely related to exact support recovery in high-dimensional statistics. Note exact support recovery using $\ell_{1}$-minimization has been intensively investigated during the last decade, see for instance $[12,19,29,32]$ and references therein. We capitalize on these works to build our estimation procedure. Observe that, for random design matrices, one often invoke the mutual incoherence property [9] to derive support recovery guarantees. Indeed, another approach relies on the Irrepresentability Condition (IC) [12,32]. Yet this approach is rather stringent and, to the best of our knowledge, proving (IC) for random matrices remains a challenging issue.

However, this article develops a new analysis of support recovery allowing to "break" the square-root bottleneck of standard procedures such as the mutual incoherence property (see Sect. 3). Interestingly, we prove that one can relax the mutual incoherence property (known to require $\mathcal{O}\left(s^{2} \log p\right)$ observations) and still ensure faithful recovery using thresholded-LASSO from $\mathcal{O}\left(s^{\alpha} \log p\right)$ observations for any $1 \leq \alpha \leq 2$.

We also mention that there exist methods that rely on additive splines and sparsity (COSSO) to perform variable selection (see [11] and references therein). In the paper, we provide a comparison, on a benchmark example, with these methods.

Recall the main goal of this paper is to draw a bridge, which, to the best of our knowledge, has not been previously drawn, between Sobol index estimation via pick-freeze estimators and sparse linear regression models. This bridge can be leveraged so as to propose an efficient estimation procedure for Sobol indices in highdimensional sparse models. 


\subsection{Organization of the paper}

The contribution of this paper is twofold: Section 2 describes a new algorithm to simultaneously estimate the Sobol indices using $\ell_{1}$-relaxation and give elementary error analysis of this algorithm (Thms. 2.2 and 2.8), and Section 3 presents a new result on exact support recovery by Thresholded-LASSO that relaxes mutual incoherence property. In particular, we prove that exact support recovery holds beyond the Welch bound.

Appendix A gives the proofs of the results in Section 2. Appendix B.1 gives preliminary results for proving Theorem 3.3 of Section 3. The remaining appendices apply these results to different designs (leading for Appendix B.2 to Thm. 3.3).

\section{A CONVEX RElaxation of Sobol's Method}

\subsection{Notation and model}

We consider the following non-parametric model. Denote by $X_{1}, \ldots, X_{p}$ the input parameters, assumed to be independent random variables of known distribution $\eta$. Let $Y$ be the model output of interest:

$$
Y=f\left(X_{1}, \ldots, X_{p}\right) \text {, }
$$

where $f: \mathbb{R}^{p} \rightarrow \mathbb{R}$ is so that $Y \in L^{2}\left(\eta^{\otimes p}\right)$ and $\operatorname{Var}(Y) \neq 0$. Assume that $f$ is additive, i.e.

$$
f\left(X_{1}, \ldots, X_{p}\right)=f_{1}\left(X_{1}\right)+\ldots+f_{p}\left(X_{p}\right)
$$

for some functions $f_{i}: \mathbb{R} \rightarrow \mathbb{R}, i=1, \ldots, p$. Note the only assumptions on the regression function $f$ are:

- $f$ is squared-integrable with respect to $\eta^{\otimes p}$;

- $f$ is additive.

In Sobol's method, we want to estimate the following vector:

$$
S=\left(S_{i}\right)_{i=1}^{p} \quad \text { where } \quad S_{i}=\frac{\operatorname{Var}\left[\mathbb{E}\left(Y \mid X_{i}\right)\right]}{\operatorname{Var}(Y)}
$$

is the $i$ th Sobol's index of $Y$ and quantifies the influence of $X_{i}$ on $Y$. In this article we present a new procedure for evaluating the Sobol's indices when $p$ is large. We make the assumption that the number of nonzero Sobol's indices:

$$
s:=\#\left\{i=1, \ldots, p \text { s.t. } S_{i} \neq 0\right\}
$$

remains small in comparison to $p$. Observe our model assumes that we know an upper bound on $s$. The Sobol's indices can be estimated using the so-called pick-freeze scheme, also know as Sobol's method [23,24]. Let $X^{\prime}$ be an independent copy of $X$ and note, for $i=1, \ldots, p$ :

$$
Y^{i}=f\left(X_{1}^{\prime}, \ldots, X_{i-1}^{\prime}, X_{i}, X_{i+1}^{\prime}, \ldots, X_{p}^{\prime}\right) .
$$

Then we have:

$$
S_{i}=\frac{\operatorname{Cov}\left(Y, Y^{i}\right)}{\operatorname{Var}(Y)}
$$

This identity leads to an empirical estimator of $S_{i}$ :

$$
\widehat{S}_{i}=\frac{\frac{1}{N} \sum Y_{k} Y_{k}^{i}-\left(\frac{1}{N} \sum \frac{Y_{k}+Y_{k}^{i}}{2}\right)^{2}}{\frac{1}{N} \sum \frac{\left(Y_{k}\right)^{2}+\left(Y_{k}^{i}\right)^{2}}{2}-\left(\frac{1}{N} \sum \frac{Y_{k}+Y_{k}^{i}}{2}\right)^{2}},
$$

where all sums are for $k$ from 1 to $N$, and $\left\{\left(Y_{k}, Y_{k}^{i}\right)\right\}_{k=1, \ldots, N}$ is an iid sample of the distribution of $\left(Y, Y^{i}\right)$ of size $N$. This estimator has been introduced in [20] and later studied in [13,16]. 
In the high-dimensional frame, the estimation of the $p$ indices using $\widehat{S}_{i}$ for $i=1, \ldots, p$ would require $(p+1) N$ evaluations of $f$ so as to generate the realizations of $\left(Y, Y^{1}, \ldots, Y^{p}\right)$. This may be too much expensive when $p$ is large and/or evaluation of $f$ is costly. Besides, thanks to our sparsity assumption, such an estimation "one variable at a time" will be inefficient, as many computations will be required to estimate zero many times. To the best of our knowledge, this paper is the first to overcome this difficulty introducing a new estimation scheme.

Remark 2.1. If $f$ is not additive, but that the non-additive part of $f$ is neglectable (in the sense that the interaction Sobol's indices are significantly lower than the principal Sobol's indices), one can consider that this non-additive part is a noise, and use the robustness properties of both Sobol's indices and the LASSO procedures we will use in the sequel to deduce that our procedure is robust with respect to small non-additive perturbations.

\subsection{Multiple pick-freeze}

We now generalize definition $(2.2)$. Let $F \subset\{1, \ldots, p\}$ be a set of indices. Define $Y^{F}$ by:

$$
Y^{F}=f\left(X^{F}\right) \quad \text { where } \quad\left(X^{F}\right)_{i}=\left\{\begin{array}{l}
X_{i} \text { if } i \in F, \\
X_{i}^{\prime} \text { if } i \in F^{c} .
\end{array}\right.
$$

where $F^{c}=\{1, \ldots, p\} \backslash F$. The name of the method stems from the fact that, to generate the $Y^{F}$ variable, all the input parameters whose indices are in $F$ are Frozen. In the pick-freeze scheme of the previous subsection, only one variable was frozen at the time, namely $F=\{i\}$. We then define:

$$
S_{F}=\frac{\operatorname{Cov}\left(Y, Y^{F}\right)}{\operatorname{Var}(Y)}
$$

which admits a natural estimator:

$$
\widehat{S}_{F}=\frac{\frac{1}{N} \sum Y_{k} Y_{k}^{F}-\left(\frac{1}{N} \sum \frac{Y_{k}+Y_{k}^{F}}{2}\right)^{2}}{\frac{1}{N} \sum \frac{\left(Y_{k}\right)^{2}+\left(Y_{k}^{F}\right)^{2}}{2}-\left(\frac{1}{N} \sum \frac{Y_{k}+Y_{k}^{F}}{2}\right)^{2}} .
$$

Under additivity hypothesis (2.1), one has:

$$
S_{F}=\sum_{i \in F} S_{i}
$$

Now, let's choose $n \in \mathbb{N}^{\star}$, subsets $F_{1}, \ldots, F_{n}$ of $\{1, \ldots, p\}$, and denote by $E$ the following vector of estimators:

$$
E=\left(\widehat{S}_{F_{1}}, \ldots, \widehat{S}_{F_{n}}\right) \text {. }
$$

Notice that, once the $F_{1}, \ldots, F_{n}$ have been chosen, the $E$ vector can be computed using $(n+1) N$ evaluations of $f$.

\subsubsection{Bernoulli regression model}

The choice of $F_{1}, \ldots, F_{n}$ can be encoded in a binary matrix $\Phi$ with $n$ lines and $p$ columns, so that:

$$
\Phi_{j i}=\left\{\begin{array}{l}
1 \text { if } i \in F_{j}, \\
0 \text { otherwise. }
\end{array} \quad j=1, \ldots, n \text { and } i=1, \ldots, p .\right.
$$

It is clear that $\left(S_{F_{1}}, \ldots, S_{F_{n}}\right)=\Phi S$, hence:

$$
E=\Phi S+\epsilon
$$


where the $\epsilon$ vector defined by $\epsilon_{j}=\widehat{S}_{F_{i}}-S_{F_{i}}$ gives the estimation error of $\Phi S$ by $E$. In practice, the $E$ vector and the $\Phi$ matrix are known, and one has to estimate $S$. Thereby, equation (2.6) can be seen as a linear regression model whose coefficients are the Sobol's indices to estimate. Moreover, observe that $n \ll p$ and $S$ sparse, hence we are in a high-dimensional sparse linear regression context. The problem (2.6) has been extensively studied in the context of sparse estimation $[1,8,19]$ and compressed sensing $[4,6]$, and a classical solution is to use the LASSO estimator [26]:

$$
\widehat{S} \in \underset{U \in \mathbb{R}^{p}}{\operatorname{argmin}}\left(\frac{1}{n}\|E-\Phi U\|_{2}^{2}+2 r\|U\|_{1}\right)
$$

where $r>0$ is a regularization parameter and:

$$
\|v\|_{2}^{2}=\sum_{j=1}^{n} v_{j}^{2}, \quad\|u\|_{1}=\sum_{i=1}^{p}\left|u_{i}\right| .
$$

Many efficient algorithms, such as LARS [10], are available in order to solve the above minimization problem, and to find an appropriate value for $r$. In high dimensional statistics, one key point for the LASSO procedure is the choice of the $\Phi$ matrix. In the Compressed Sensing and high-dimensional statistics (see for instance [1-7] and references therein), a random matrix with i.i.d. coefficients often proves to be a good choice, hence we will study possible random choices for $\Phi$.

Input: $f$ (regression function), $N$ (Monte-Carlo sample size), $n$ (number of estimations) and $r$ (regularization parameter).

Output: Estimation $\widehat{S}$ of the Sobol's indices $S$.

(1) Randomly sample a 0-1 matrix $\Phi$ with Bernoulli distribution of parameter $\mu$.

(2) Deduce from $\Phi$ the $F_{1}, \ldots, F_{n}$ subsets using (2.5).

(3) Generate a $N$-sized sample of $\left(Y, Y^{F_{1}}, \ldots, Y^{F_{n}}\right)$.

(4) Use this sample in (2.3), for $F=F_{1}, \ldots, F_{n}$, to obtain the $E$ vector (2.4).

(5) Solve problem (2.7) to obtain an $\widehat{S}$ which estimates $S$.

Algorithm 1: Summary of the method "Randomized Pick-Freeze" (RPF) for Bernoulli matrices.

Given the binary constraint on $\Phi$, we will choose a Bernoulli distribution with parameter $\mu \in] 0 ; 1[$. In this model, $\left(\Phi_{j i}\right)_{j, i}$ are independent, with for all $i, j$ :

$$
\mathbb{P}\left(\Phi_{j i}=1\right)=\mu=1-\mathbb{P}\left(\Phi_{j i}=0\right) .
$$

Theorem 2.2 ( $\ell^{\infty}$ error bound). Suppose that:

(1) $\delta$ is a real in $] 0 ; \frac{1-\mu}{16 s}[$;

(2) $\epsilon$ is a centered Gaussian vector whose covariance matrix has $\sigma^{2}$ as largest eigenvalue;

(3) $r=A \sigma \sqrt{\mu(1+\delta)} \sqrt{\frac{\ln p}{n}}$ for some $A>2 \sqrt{2}$.

Let:

$$
\begin{aligned}
t= & \left(\frac{3}{2}+\frac{24(\mu+\delta)}{\frac{1-\mu}{s}-16 \delta}\right) \frac{r}{\mu} \\
\alpha= & 1-\left(1-p^{1-A^{2} / 8}\right)\left(1-2 \exp \left(-2 n \delta^{2} \mu^{2}+\ln p\right)\right) \\
& +\exp \left(-2 n \delta^{2} \mu^{2}+2 \ln p\right)
\end{aligned}
$$


Then, with probability at least $1-\alpha$, any solution $\widehat{S}$ of (2.7) satisfies:

$$
\max _{i=1, \ldots, p}\left|\widehat{S}_{i}-S_{i}\right| \leq t
$$

Proof. A proof can be found in Appendix A.1.

Remark 2.3. For the probability above to be greater than zero, it is necessary to have:

$$
n \geq \frac{\ln p}{\delta^{2} \mu^{2}} \geq \frac{256 s^{2} \ln p}{\mu^{2}(1-\mu)^{2}}
$$

Remark 2.4. The statement of Theorem 2.2 can be compared to standard results in high-dimensional statistics such as exact support recovery under mutual incoherence property [19]. Nevertheless, observe that a standard assumption is that the column norm of the design matrix is $\sqrt{n}$ while in our frame this norm is random with expectation of order $\sqrt{\mu n}$.

Remark 2.5. In our context, the second hypothesis of the above theorem does not exactly hold; indeed, $\epsilon$ is only asymptotically Gaussian (when $N \rightarrow+\infty$ ), see [16] for instance. However, for practical purposes, the observed convergence is fast enough. One can also see a related remark in our proof of this theorem, in Appendix A.1.

Corollary 2.6 (Support recovery by Thresholded-LASSO). Let:

$$
S_{\min }=\min _{\substack{i=1, \ldots, p \\ \text { s.t. } S_{i} \neq 0}} S_{i}
$$

Then, under the same assumptions of Theorem 2.2, we have, with probability greater than $1-\alpha$ and for all $i=1, \ldots, p$ :

$$
\widehat{S}_{i}>t \Longrightarrow S_{i}>0
$$

and:

$$
\widehat{S}_{i}<S_{\min }-t \Longrightarrow S_{i}=0
$$

Proof of Corollary 2.6. For the first point, notice that:

$$
\left|S_{i}\right| \geq\left|\widehat{S}_{i}\right|-\left|\widehat{S}_{i}-S_{i}\right| \geq\left|\widehat{S}_{i}\right|-t>0 \text { if } \widehat{S}_{i}>t .
$$

For the second point: if $\widehat{S}_{i}<S_{\min }-t$, we have:

$$
\left|S_{i}\right| \leq\left|S_{i}-\widehat{S}_{i}\right|+\left|\widehat{S}_{i}\right|<t+\left(S_{\min }-t\right)=S_{\min }
$$

and $S_{i}=0$ by definition of $S_{\min }$.

Remark 2.7 (important). Theorem 2.2 and Corollary 2.6 show that one can identify the most important inputs of a function as soon as the corresponding Sobol indices are above the threshold $t$. Recall Thresholded-LASSO is a thresholded version of any solution to (2.7). Moreover, observe that we do not address the issue of estimating the Sobol's indices. This can be done using a two-step procedure: estimate the support using Thresholed-LASSO and then estimate the Sobol indices using a standard least squares estimator. 


\subsubsection{Rademacher regression model}

The choice of $F_{1}, \ldots, F_{n}$ can also be encoded in a \pm 1 matrix $\Phi$ with $n$ lines and $p$ columns, so that:

$$
\Phi_{j i}=\left\{\begin{array}{ll}
1 \quad \text { if } i \in F_{j}, \\
-1 & \text { otherwise. }
\end{array} \quad j=1, \ldots, n \text { and } i=1, \ldots, p .\right.
$$

It is clear that:

$$
\left(S_{F_{1}}^{\Delta}, \ldots, S_{F_{n}}^{\Delta}\right)=\Phi S
$$

where $S_{F_{i}}^{\Delta}=S_{F_{i}}-S_{F_{i}^{c}}$. Hence:

$$
E=\Phi S+\epsilon^{\Delta},
$$

where the $\epsilon$ vector defined by $\epsilon_{j}^{\Delta}=\widehat{S}_{F_{i}}^{\Delta}-S_{F_{i}}^{\Delta}$ gives the estimation error of $\Phi S$ by $E$. Thus, the problem of estimating $S$ from $E$ has been casted into linear regression which can be tackled by (2.7).

Input: $f$ (regression function), $N$ (Monte-Carlo sample size), $n$ (number of estimations) and $r$ (regularization parameter).

Output: Estimation $\widehat{S}$ of the Sobol's indices $S$.

(1) Sample a $\Phi$ matrix according to a \pm 1 symmetric Rademacher distribution.

(2) Deduce from $\Phi$ the $F_{1}, \ldots, F_{n}$ subsets using the correspondance (2.10).

(3) Generate a $N$-sized sample of $\left(Y, Y^{F_{1}}, \ldots, Y^{F_{n}}\right)$.

(4) Use this sample in (2.3), for $F=F_{1}, \ldots, F_{n}$, to obtain the $E$ vector (2.4).

(5) Solve problem (2.7) to obtain an $\widehat{S}$ which estimates $S$.

Algorithm 2: Summary of the method "Randomized Pick-Freeze" (RPF) for Rademacher matrices.

We now consider a different sampling procedure for $\Phi$, which will make it possible to improve on the constants in (2.9) as it will be stated in (2.13). Specifically, we sample $\Phi$ using a symmetric Rademacher distribution:

$$
\left(\Phi_{j i}\right)_{j, i} \text { are independent : } P\left(\Phi_{j i}=1\right)=P\left(\Phi_{j i}=-1\right)=\frac{1}{2} .
$$

The following theorem is the equivalent of Theorem 2.2 for Rademacher designs.

Theorem 2.8 ( $\ell^{\infty}$ error bound). Suppose that:

(1) $\epsilon$ is a centered Gaussian vector whose covariance matrix has $\sigma^{2}$ as largest eigenvalue;

(2) $\delta=\frac{1}{7 \delta^{\prime} s}$ for some real $\delta^{\prime}>1$;

(3) $r=A \sigma \sqrt{\frac{\ln p}{n}}$ for some $A>2 \sqrt{2}$.

Let:

$$
\begin{aligned}
t & =\frac{3}{2}\left(1+\frac{16}{5\left(\delta^{\prime}-1\right)}\right) r \\
\alpha & =1-\left(1-p^{1-A^{2} / 8}\right)\left(1-\exp \left(-n \frac{49 \delta^{2} s^{2}}{2}+2 \ln p\right)\right) .
\end{aligned}
$$

Then, with probability at least $1-\alpha$, any solution $\widehat{S}$ of (2.7) satisfies:

$$
\max _{i=1, \ldots, p}\left|\widehat{S}_{i}-S_{i}\right| \leq t .
$$




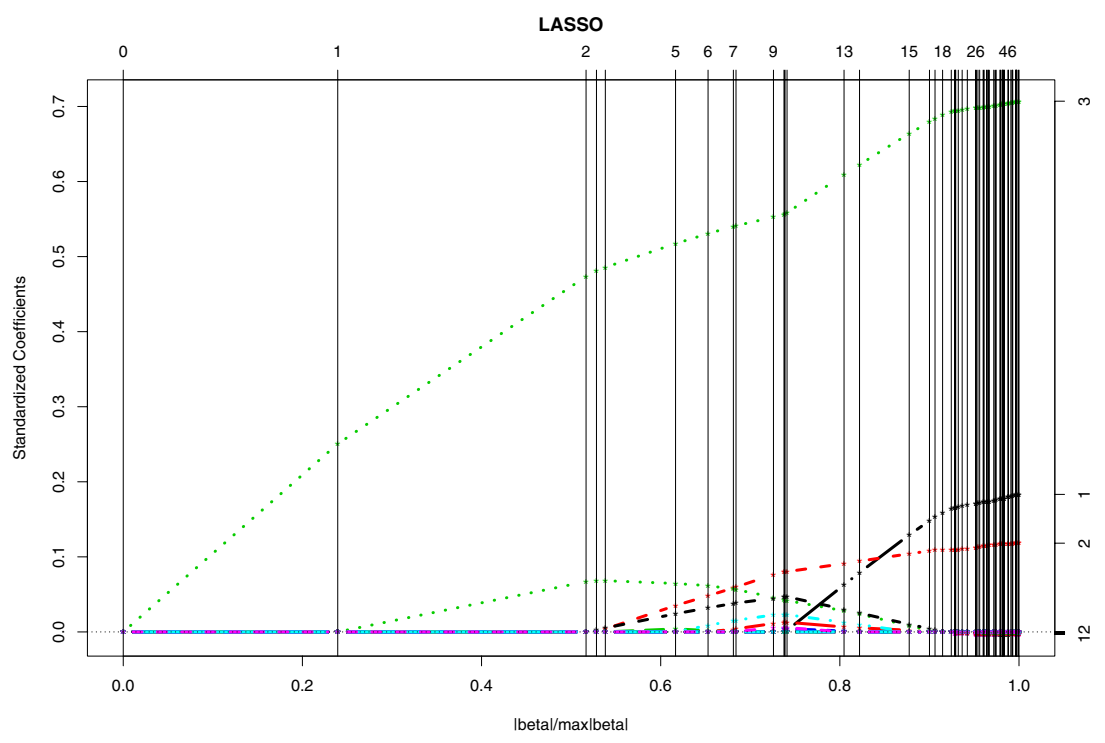

FiguRE 1. LASSO convergence path for a Bernoulli design.

Proof. A proof can be found in Appendix A.2.

Remark 2.9. For the probability above to be greater than zero, it is necessary to have:

$$
n \geq C s^{2} \ln p
$$

for some constant $C>0$.

Remark 2.10. Support recovery property (Cor. 2.6) also holds in this context.

Remark 2.11. We see that there are many simulation parameters $(n, N, r, A, \delta)$ to choose. The point is to balance between the precision of the obtained confidence interval $(t)$ and the level of this confidence interval $(\alpha)$. The "adequate" choice heavily depends on the case at hand (the $f$ function, which dictates $\sigma, s$, and $p$ ), as well as the precision/confidence requirements, and the constraints on computational budget (dictated by $N, n$, and the run time for $f$ ). The adequate tuning of these parameters should be left to the practitioner, which will typically prescribe $t$ and $\alpha$, find a rough estimate of $\sigma$ and $s$, and then find $n, N, \delta, r$ and $A$ to satisfy its prescriptions on $\mathrm{t}$ and alpha.

\subsection{Numerical experiments}

\subsubsection{LASSO convergence paths}

In this section, we perform a numerical test of the "Randomized Pick-Freeze" estimation procedure for Bernoulli and Rademacher matrices, summarized respectively on the 5th and 7th page. We use the following model:

$$
Y=f\left(X_{1}, \ldots, X_{300}\right)=X_{1}^{2}+4 X_{1}+4 X_{2}+10 X_{3},
$$

hence $p=300$ and $s=3$, with $\left(X_{i}\right)_{i=1, \ldots, 300}$ iid uniform on $[0,1]$. It is easy to see that, in this model, we have $S_{3}>S_{1}>S_{2}>0$ and $S_{i}=0$ for all $i>3$; more precisely, $S_{1}=0.18, S_{2}=0.11, S_{3}=0.71$. The tests are performed by using $n=30$. The obtained LASSO regularization paths (ie., the estimated indices for different choices of the penalization parameter $r$ ) are plotted in Figure 1 (for Bernoulli design matrix with parameter 


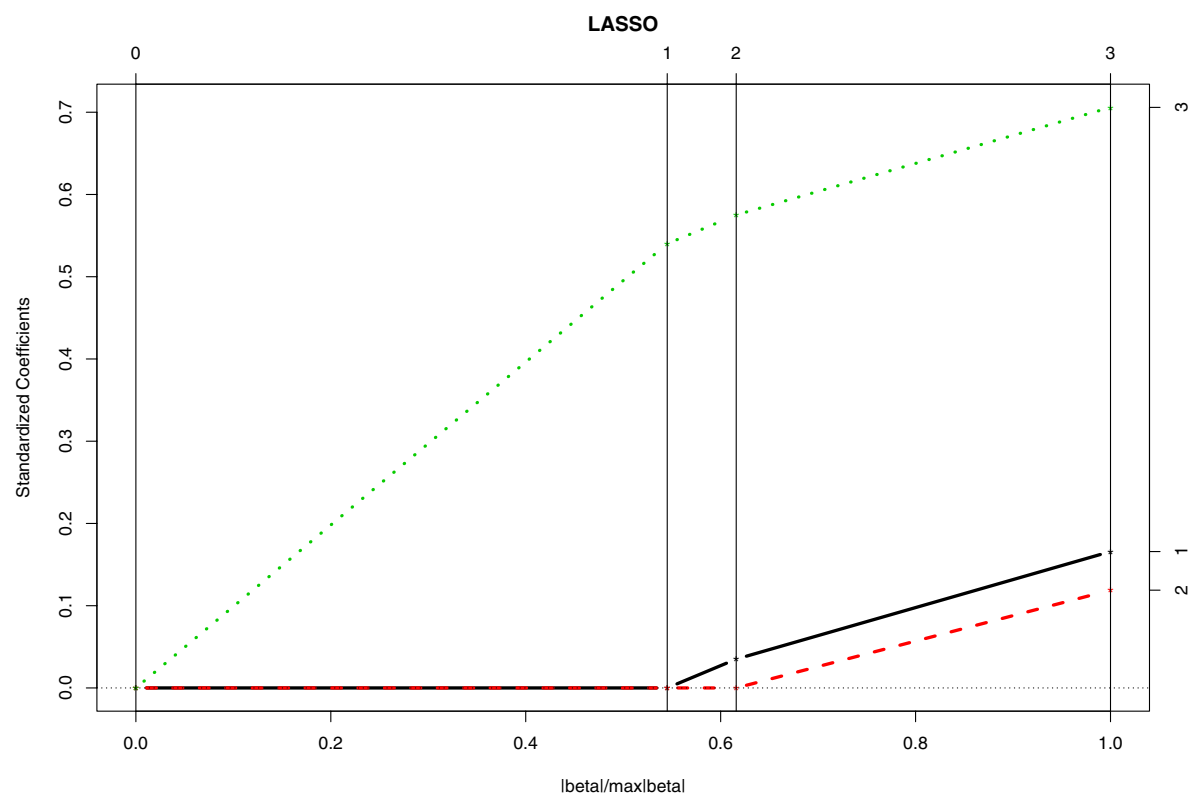

FiguRE 2. LASSO convergence path for a Rademacher design.

$\mu=1 / 2$ ) and Figure 2 (for Rademacher design matrix). The Monte-Carlo sample size used are $N=3000$ and $N=2000$, respectively for Bernoulli and Rademacher designs. This difference in sample sizes accounts for the increase in the number of required evaluations of the $f$ function when a Rademacher design is used (as, in this case, each replication is a difference of two pick-freeze estimators on the same design).

We observe that the Rademacher design seems to perform better (as LASSO convergence is faster) than the Bernoulli design, in accordance with the remarks made in the beginning of Section 3. Both designs perfectly recover the active variables (the support of $S$ ), as well as the ordering of indices. Note that the proposed method requires only $30 \times 2 \times 3000=180000$ evaluations of the $f$ function to estimate the 300 Sobol indices, while a classic one-by-one pick-freeze estimation with the same Monte-Carlo sample size would require $3000 \times(300+1)=$ 903000 evaluations of $f$.

\subsubsection{Second illustration}

We now take a second example, taken from [11]:

$$
\begin{aligned}
Y= & 5 X_{1}+3\left(2 X_{2}-1\right)^{2}+4 \frac{\sin \left(2 \pi X_{3}\right)}{2-\sin \left(2 \pi X_{3}\right)}+6\left(0.1 \sin \left(2 \pi X_{4}\right)+0.2 \cos \left(2 \pi X_{4}\right)\right. \\
& +0.3 \sin \left(2 \pi X_{4}\right)^{2}+0.4 \cos \left(2 \pi X_{4}\right)^{3}+0.5 \sin \left(2 \pi X_{4}\right)^{3}
\end{aligned}
$$

with $p=1000$, and $X_{i}$ uniform on $[0 ; 1]$.

The true values of the Sobol's indices are:

$$
S_{1}=0.13, S_{2}=0.05, S_{3}=0.21, S_{4}=0.6 .
$$

We take: (for Bernoulli design) $n=30$ and $N=400$, and (for Rademacher design) $n=30, N=266$. The resulting LASSO convergence paths are given in Figures 3 (for Bernoulli design) and 4 (for Rademacher design).

The results are, again, quite satisfactory, from both qualitative (support recovery, ordering of indices) and quantitative (the estimated values are close to the true values) points of view. This computation required 24000 


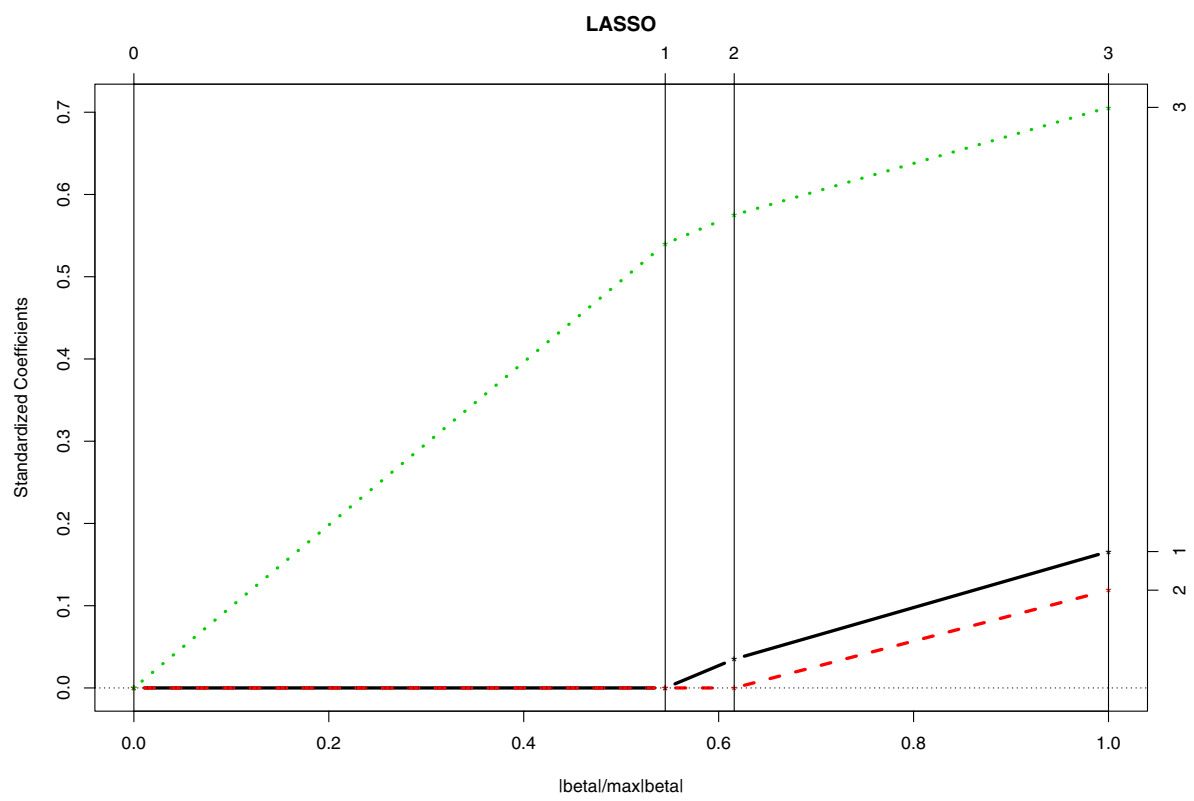

FiguRE 3. LASSO convergence path for a Rademacher design.

calls to the $f$ function. In [11], the authors have performed support recovery with 400 calls to $f$, using splinebased nonparametric independence screening (NIS) methods. Hence, our method require a little more runs in this case but, one should keep in mind that our method does not assume that the $f$ function has some regularity (i.e., approachability by splines). Besides, the overall computational time needed to produce the support estimation is much smaller for our method. This can be explained by the fact that we only require a single LASSO to produce our estimators, in contrast to the NIS methods [11] which may have a greater complexity. We also note that our method does not only perform support recovery, it also gives a robust quantification of the importance of each input variable. To finish, we mention that, if the $f$ function is regular and only a small sample of evaluations of $f$ is available, it is still possible to use our estimation method using a large sample of evaluations of a smoothed version of $f$ ("metamodel"), estimated with a COSSO method from a small sample of evaluations of $f$.

\subsubsection{Illustration of $\ell^{\infty}$ error bounds}

We now present a synthetic example which shows the performance of the Rademacher RPF algorithm, used with Theorem 2.8 and the support recovery corollary.

Suppose that we work on a model with $p=30000$ inputs, with only $s=3$ of them have a nonzero Sobol's index. We postulate that all the $\widehat{S}_{i}$ estimators, as well as the $\widehat{S}_{F}^{\Delta}$ have standard Gaussian distribution. By using $N=10^{6}$ and $n=100$ in Theorem 2.8, we get that the $t$ error bound given in this theorem is $t=0.03$, with probability greater than $1-\alpha=95 \%$. Hence, by doing calling $3 N n=3 \times 10^{8}$ to the $f$ function, one can correctly identify parameters whose Sobol's indices are greater than 0.03 .

On the other hand, when using classical one-by-one Sobol index estimation, one has to perform $p=30000$ independent estimations of Sobol index confidence intervals, at level $1-0.95^{1 / 30000}=1.71 \times 10^{-6}$ (by using ÅăidÃa̧k correction). From the quantiles of the Gaussian distribution, the length of these intervals is $9.568 / \sqrt{N}$. Hence, getting confidence intervals of width 0.03 require $N^{\prime}=(9.568 / 0.03)^{2} \approx 101720$ sample size. Hence, the total cost for this method is $2 N^{\prime}(p+1)=6103200000 \approx 61 \times 10^{8}$ calls to the $f$ function. 


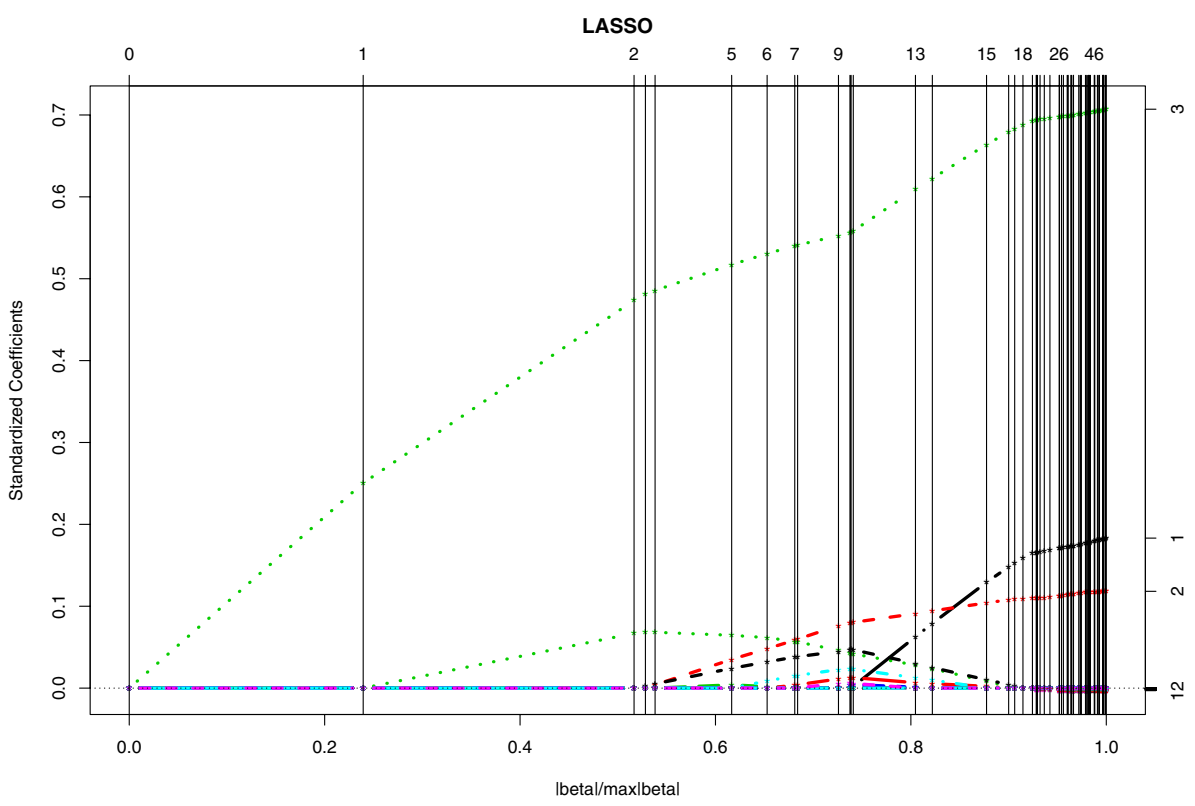

FIgURE 4. LASSO convergence path for a Bernoulli design.

\section{BREAKING THE SQUARE-ROOT BOtTLENECK}

\subsection{Relaxation of the mutual incoherence property}

In the beginning of this paper, we have showed results that are limited by the constraint $n \geq C s^{2} \log p$ for some constant $C$. This limitation is due to the use of the mutual incoherence property in the proofs, which is heuristically bounded by Welch's bound [31]. We now present a new approach, based on Universal Distortion Property [8] and a relaxed version of the coherence which enables to break this "bottleneck" for a large set of random design matrices encompassing Rademacher designs.

Theorem 3.1 (Exact support recovery with Thresholded-LASSO). Assume that for all $\gamma \in \mathbb{R}^{p}$, for all $T \subseteq$ $\{1, \ldots, p\}$ such that $|T| \leq s$,

$$
\left\|\gamma_{T}\right\|_{1} \leq \rho \sqrt{s}\|\Phi \gamma\|_{2}+\kappa\|\gamma\|_{1}
$$

where $\rho>0$ and $1 / 2>\kappa>0$. Moreover, assume that:

$$
\max _{1 \leq k \neq l \leq p} \frac{1}{n}\left|\sum_{j=1}^{n} \Phi_{j, k} \Phi_{j, l}\right| \leq \theta_{1} \quad \text { and } \quad \forall i, \frac{1}{n}\left\|\Phi_{i}\right\|_{2}^{2} \geq \theta_{2},
$$

where $\Phi_{i}$ denotes the columns of $\Phi$. Let $r_{0}>0$ and suppose that the regularizing parameter $r$ of the convex program (2.7) enjoys:

$$
r>\frac{r_{0}}{1-2 \kappa} .
$$

Then, on the event $\left\{(1 / n)\left\|\Phi^{\top} \epsilon\right\|_{\infty} \leq r_{0}\right\}$, any solution $\hat{S}$ to (2.7) satisfies:

$$
\|\hat{S}-S\|_{\infty} \leq \frac{1}{\theta_{2}}\left[1+\frac{r_{0}}{r}+\frac{2 n \theta_{1} \rho^{2} s}{1-\left(r_{0} / r\right)-2 \kappa}\right] r .
$$


Proof. The first order optimality conditions of the convex program (2.7) shows that there exists $\tau \in \mathbb{R}^{p}$ such that $\|\tau\|_{\infty} \leq 1$ and:

$$
\frac{1}{n} \Phi^{\top}(E-\Phi \hat{S})=r \tau
$$

Set $\Delta=\hat{S}-S$ and $\Psi=(1 / n) \Phi^{\top} \Phi$. We assume that $(1 / n)\left\|\Phi^{\top} \epsilon\right\|_{\infty} \leq r_{0}$. It holds:

$$
\|\Psi \Delta\|_{\infty} \leq r+r_{0} .
$$

Moreover, Lemma B.2 shows that:

$$
\|\Delta\|_{1} \leq \frac{2 n r \rho^{2} s}{1-r_{0} / r-2 \kappa} .
$$

Since each entry in the matrix $\Psi-\operatorname{Diag}\left(\left\|\Phi_{1}\right\|_{2}^{2} / n, \ldots,\left\|\Phi_{p}\right\|_{2}^{2} / n\right)$ is less than $\theta_{1}$, we deduce that:

$$
\begin{aligned}
\theta_{2}\|\Delta\|_{\infty} & \leq\|\Psi \Delta\|_{\infty}+\left\|\left(\Psi-\operatorname{Diag}\left(\left\|\Phi_{1}\right\|_{2}^{2} / n, \ldots,\left\|\Phi_{p}\right\|_{2}^{2} / n\right)\right) \Delta\right\|_{\infty}, \\
& \leq r+r_{0}+\theta_{1}\|\Delta\|_{1} \\
& \leq\left[1+\frac{r_{0}}{r}+\frac{2 n \theta_{1} \rho^{2} s}{1-r_{0} / r-2 \kappa}\right] r
\end{aligned}
$$

using (3.3) and (3.4).

Remark 3.2. Observe that (3.1) is the Universal Distortion Property (UDP) appearing in the article [8]. This property is the weakest property to obtain oracle inequalities with LASSO, see [8]. In particular, Restricted Isometry Property (RIP) [5], Restricted Eigenvalue Condition (REC) [2], Compatibility Condition (CC) [30], or $H_{s, 1}$ condition [17] imply UDP. Essentially, this shows that all random matrices considered in high-dimensional statistics fit the condition (3.1).

Furthermore, Condition (3.2) is a relaxed version of the mutual incoherence property [9] that requires:

$$
\max _{1 \leq k \neq l \leq p}\left|\sum_{j=1}^{n} \Phi_{j, k} \Phi_{j, l}\right| \leq \frac{1}{2 s-1} \min _{i=1, \ldots, p}\left\|\Phi_{i}\right\|_{2}^{2},
$$

where $\Phi_{i}$ denotes the columns of $\Phi$. Using Welch's bound [31], one can prove that this condition implies $n \geq$ $C s^{2} \log p$ for some constant $C>0$. Note that Theorem 3.1 overcomes this difficulty allowing larger upper bounds on the correlations $\max _{1 \leq k \neq l \leq p}\left|\sum_{j=1}^{n} \Phi_{j, k} \Phi_{j, l}\right|$. As done in the next section, this result allows to derive support recovery guarantees from $\mathcal{O}\left(s^{\alpha} \log (p)\right)$ observations for any value $1 \leq \alpha \leq 2$.

\subsection{Rademacher designs}

In this section we focus on Rademacher designs defined by $(2.12)$, namely $\left(\Phi_{j i}\right)_{j, i}$ are independent and for all $i, j$, it holds $\mathbb{P}\left(\Phi_{j i}= \pm 1\right)=1 / 2$.

Theorem 3.3 (Exact recovery with Rademacher designs). There exists universal constants $C_{1}, C_{2}, C_{3}>0$ such that the following holds. Let $c>1$ and $\Phi \in\{ \pm 1\}^{n \times p}$ a Rademacher matrix drawn according to (2.12) with:

- $n \geq n_{0}:=C_{1} s \log \left(C_{2} p\right)$;

- $s \geq 6(2+c) / C_{1}$;

- $\epsilon \sim \mathcal{N}\left(0, \Sigma_{n}\right)$ and the covariance diagonal entries enjoy $\Sigma_{i, i} \leq \sigma^{2}$. 
Let $\hat{S}$ be any solution to (2.7) with regularizing parameter:

$$
r \geq r_{1}:=45 \sigma\left[\frac{c \log p}{n}\right]^{1 / 2},
$$

Then, with a probability greater than $1-3 p^{-c}-2 \exp \left(-C_{3} n\right)$,

$$
\|\hat{S}-S\|_{\infty} \leq \sigma \sqrt{\frac{n_{0}}{n}}\left[\frac{r}{r_{1}}\right]\left[C_{1}^{\prime}+\frac{C_{2}^{\prime}}{\sqrt{s}}\right] \sqrt{s}
$$

where $C_{1}^{\prime}=35869(c(2+c))^{1 / 2} / C_{1}$ and $C_{2}^{\prime}=46.31 c^{1 / 2} / C_{1}^{1 / 2}$.

Proof.

- Invoke Lemma B.5 to get that:

$$
\max _{1 \leq k \neq l \leq p} \frac{1}{n}\left|\sum_{j=1}^{n} \Phi_{j, k} \Phi_{j, l}\right| \leq\left[\frac{(2+c) 8}{3 C_{1}}\right]^{1 / 2} \frac{1}{\sqrt{s}},
$$

with probability greater than $1-2 p^{-c}$.

- Set $r_{0}:=\sigma(2 c \log p / n)^{1 / 2}$ and $Z_{i}=(1 / n) \Phi^{\top} \epsilon$. Observe that $Z_{i}$ is centered Gaussian random variable with variance less than $\sigma^{2} / n$. Taking union bounds, it holds:

$$
\mathbb{P}\left[(1 / n)\left\|\Phi^{\top} \epsilon\right\|_{\infty}>r_{0}\right] \leq \sum_{i=1}^{p} \mathbb{P}\left[\left|Z_{i}\right|>\sqrt{2 c} \sqrt{\log p} \sigma / \sqrt{n}\right] \leq p^{1-c},
$$

using $\left\|\Phi_{i}\right\|_{2}^{2}=n$ and the fact that, for $\sqrt{2 c} \sqrt{\log p} \geq \sqrt{2 \log 2}$, we have:

$$
\mathbb{P}[|\mathcal{N}(0,1)|>\sqrt{2 c} \sqrt{\log p}] \leq \frac{1}{\sqrt{\pi \log 2}} \exp (-c \log p) \leq p^{-c} .
$$

- From Lemma B.3 and Lemma B.4 with $\delta=9 / 50$ and $\kappa=4 / 9$, it holds that, with a probability greater than $1-2 \exp \left(-C_{3} n\right)$, for all $\gamma \in \mathbb{R}^{p}$ and for $T \subseteq\{1, \ldots, p\}$ such that $|T| \leq s$,

$$
\left\|\gamma_{T}\right\|_{1} \leq 4.4128\left(\frac{s}{n}\right)^{1 / 2}\|\Phi \gamma\|_{2}+\frac{4}{9}\|\gamma\|_{1} .
$$

Observe that $C_{1}=5 /\left(c_{1} \delta^{2}\right), C_{2}=c_{2} / \delta^{2}$ and $C_{3}=c_{3} C_{1}$ where $c_{1}, c_{2}, c_{3}$ are universal constants appearing in Lemma B.3 and $\delta=9 / 50$.

- Invoke Theorem 3.1 with parameters $\rho=4.4128 / \sqrt{n}, \kappa=4 / 9, \theta_{2}=1$ and $\theta_{1}=\left((2+c) 8 /\left(3 C_{1} s\right)\right)^{1 / 2}$, to get that for all regularizing parameter $r \geq r_{1}:=31.74 r_{0}$,

$$
\|\hat{S}-S\|_{\infty} \leq\left(1.0316+799\left(\frac{2+c}{C_{1}}\right)^{1 / 2} \sqrt{s}\right) r,
$$

on the event $\left\{(1 / n)\left\|\Phi^{\top} \epsilon\right\|_{\infty} \leq r_{0}\right\}$.

Remark 3.4. Observe that (3.5) reads:

$$
\|\hat{S}-S\|_{\infty} \leq\left[\frac{r}{r_{1}}\right]\left[C_{1}^{\prime}+\frac{C_{2}^{\prime}}{\sqrt{s}}\right] \sigma \sqrt{\frac{C_{1} s^{2} \log \left(C_{2} p\right)}{n}},
$$

where $C_{1}, C_{2}, C_{1}^{\prime}, C_{2}^{\prime}>0$ are constants. It shows that, for all $\alpha>0$, Thresholded-LASSO exactly recovers the true support if the non-zero coefficients are above a threshold that is proportional to $\sigma s^{\frac{1-\alpha}{2}}$ from $n=\mathcal{O}\left(s^{1+\alpha} \log p\right)$ observations. Hence, we have tackled the regime $0<\alpha<1$ where the elementary analysis of Theorem 2.8 fails to be applicable. 


\section{Conclusions}

We have presented a new and performant method for estimating Sobol's indices in high-dimensional additive models. We have shown that this method can lead to very good results in terms of computational costs. Besides, the error analysis of our algorithm led us to propose the results in Section 3, which are also of interest outside of the Sobol's indices context, and which gives support recovery property for thresholded LASSO that are, to our best knowledge, greatly improving the results of the literature.

\section{Appendix A. Proof of the theorems}

\section{A.1. Proof of Theorem 2.2}

We capitalize on $[3,19,32]$ to prove sup-norm error bound when the design matrix has Bernoulli distribution.

\section{Step 1: Rescaling.}

We rewrite $(2.6)$ as $\tilde{E}=\tilde{\Phi} S+\tilde{\epsilon}$ where:

$$
\tilde{E}=\frac{1}{\sqrt{\mu}} E, \quad \tilde{\Phi}=\frac{1}{\sqrt{\mu}} \Phi, \quad \tilde{\epsilon}=\frac{1}{\sqrt{\mu}} \epsilon
$$

Note $\widehat{S}$ satisfies:

$$
\widehat{S} \in \underset{U \in \mathbb{R}^{p}}{\operatorname{argmin}}\left\{\frac{1}{n}\|\tilde{E}-\tilde{\Phi} U\|_{2}^{2}+2 \tilde{r}\|U\|_{1}\right\}
$$

with:

$$
\tilde{r}=\frac{r}{\mu}=A \sigma \sqrt{\frac{1+\delta}{\mu}} \sqrt{\frac{\ln p}{n}} .
$$

\section{Step 2: Expectation and concentration.}

We define:

$$
\Psi=\frac{1}{n} \tilde{\Phi}^{T} \tilde{\Phi}=\frac{1}{n \mu} \Phi^{T} \Phi .
$$

Thanks to the rescaling above, we have, for all $i=1, \ldots, p$ :

$$
\mathbb{E}\left(\Psi_{i i}\right)=\frac{1}{n} \sum_{k=1}^{n} \mathbb{E}\left(\tilde{\Phi}_{k i}^{2}\right)=1,
$$

and, for all $j=1, \ldots, p, j \neq i$ :

$$
\mathbb{E}\left(\Psi_{i j}\right)=\frac{1}{n} \sum_{k=1}^{n} \mathbb{E}\left(\tilde{\Phi}_{k i} \tilde{\Phi}_{k j}\right)=\mu .
$$

Besides, Hoeffding's inequality [15] gives that for all $i=1, \ldots, p$ and any $\delta>0$,

$$
\mathbb{P}\left(\left|\Psi_{i i}-1\right| \geq \delta\right)=\mathbb{P}\left(\left|\frac{1}{n} \sum_{k=1}^{n}\left(\Phi_{k i}^{2}-\mu\right)\right| \geq \delta \mu\right) \leq 2 \exp \left(-2 n \delta^{2} \mu^{2}\right),
$$

and, similarly, for any $j \neq i$,

$$
\mathbb{P}\left(\left|\Psi_{i j}-\mu\right| \geq \delta\right) \leq 2 \exp \left(-2 n \delta^{2} \mu^{2}\right)
$$

Thus, by union bound:

$$
\mathbb{P}\left(\max _{i=1, \ldots, p}\left|\Psi_{i i}-1\right| \geq \delta\right) \leq 2 \exp \left(-2 n \delta^{2} \mu^{2}+\ln p\right)
$$


and:

$$
\begin{aligned}
\mathbb{P}\left(\max _{j \neq i}\left|\Psi_{i j}-\mu\right| \geq \delta\right) & \leq 2 \exp \left(-2 n \delta^{2} \mu^{2}+\ln \frac{p(p-1)}{2}\right) \\
& \leq \exp \left(-2 n \delta^{2} \mu^{2}+2 \ln p\right) .
\end{aligned}
$$

\section{Step 3: Noise control.}

We proceed as in the proof of (Lem. 1 of [19]). We define, for $i=1, \ldots, p$ :

$$
Z_{i}=\frac{1}{n} \sum_{j=1}^{n} \tilde{\Phi}_{j i} \tilde{\epsilon}_{j}=\frac{1}{n}\left(\tilde{\Phi}^{T} \tilde{\epsilon}\right)_{i} .
$$

We define the following event:

$$
\mathcal{B}=\left\{\max _{i=1, \ldots, p}\left|\Psi_{i i}-1\right| \leq \delta\right\} .
$$

For a given $\Phi$, we denote by $\Sigma=\Sigma(\Phi)$ the covariance matrix of $\epsilon$, hence the covariance matrix of $\tilde{\epsilon}$ is $\Sigma / \mu$. Note that, as a function of $\Phi, \Sigma$ is also a random variable. We also denote by $\operatorname{Var} Z_{i}$ the variance of $Z_{i}$ for a fixed $\Phi$, which is also a $\Phi$-mesurable random variable. Conditionally on $\mathcal{B}$, we have:

$$
\begin{aligned}
\operatorname{Var} Z_{i} & =\frac{1}{n^{2}} \operatorname{Var}\left[\left(\tilde{\Phi}^{T} \tilde{\epsilon}\right)_{i}\right] \\
& =\frac{1}{\mu n^{2}} e_{i}^{T}\left(\tilde{\Phi}^{T} \Sigma \tilde{\Phi}\right) e_{i} \text { where }\left(e_{i}\right)_{k}=\left\{\begin{array}{l}
1 \text { if } i=k \\
0 \text { else }
\end{array}\right. \\
& =\frac{1}{\mu n^{2}}\left(\tilde{\Phi} e_{i}\right)^{T} \Sigma\left(\tilde{\Phi} e_{i}\right) \\
& \leq \frac{1}{\mu n^{2}} \sigma^{2}\left\|\tilde{\Phi} e_{i}\right\|_{2}^{2} \\
& =\frac{1}{n \mu} \sigma^{2} e_{i}^{T} \Psi e_{i} \\
& \leq \frac{1}{n \mu} \sigma^{2}(1+\delta) \text { as } \mathcal{B} \text { holds. }
\end{aligned}
$$

Now consider the following event:

$$
\mathcal{A}=\bigcap_{i=1}^{p}\left\{\left|Z_{i}\right| \leq \frac{\tilde{r}}{2}\right\} .
$$

We have:

$$
\mathbb{P}(\mathcal{A} \cap \mathcal{B})=\mathbb{P}(\mathcal{A} \mid \mathcal{B}) \mathbb{P}(\mathcal{B})
$$

From union bound and standard results on Gaussian tails, we get:

$$
\begin{aligned}
\mathbb{P}(\mathcal{A} \mid \mathcal{B}) & \geq 1-p \exp \left(-\frac{n \mu}{2 \sigma^{2}(1+\delta)}\left(\frac{\tilde{r}}{2}\right)^{2}\right) \\
& \geq 1-p^{1-\frac{A^{2}}{8}}
\end{aligned}
$$

by using (A.1). Hence, Step 2 gives:

$$
\mathbb{P}(\mathcal{A} \cap \mathcal{B}) \geq\left(1-p^{1-A^{2} / 8}\right)\left(1-2 \exp \left(-2 n \delta^{2} \mu^{2}+\ln p\right)\right) .
$$


Remark A.1. following Remark 2.5 (given after the statement of the proven theorem), one can precisely account for the non-gaussianity of the $\epsilon$ noise by subtracting a correction term to minor $\mathbb{P}(\mathcal{A} \mid \mathcal{B})$, by using the Berry-Esseen's theorem for the $\hat{S}$ estimator given in [13].

Now suppose that $\mathcal{A} \cap \mathcal{B}$ is realized. We have:

$$
\frac{1}{n}\left\|\tilde{\Phi}^{T} \tilde{\epsilon}\right\|_{\infty} \leq \frac{\tilde{r}}{2}
$$

where

$$
\|v\|_{\infty}=\max \left|v_{i}\right|
$$

Set $\Delta=S-\widehat{S}$. We have:

$$
\begin{aligned}
\|\Psi \Delta\|_{\infty} & =\frac{1}{n}\left\|\tilde{\Phi}^{T} \tilde{\Phi} \Delta\right\|_{\infty}, \\
& =\frac{1}{n}\left\|\tilde{\Phi}^{T} \tilde{\Phi} S-\tilde{\Phi}^{T} \tilde{\Phi} \widehat{S}\right\|_{\infty}, \\
& =\frac{1}{n}\left\|\tilde{\Phi}^{T} \tilde{E}-\tilde{\Phi}^{T} \tilde{\epsilon}-\tilde{\Phi}^{T} \tilde{\Phi} \widehat{S}\right\|_{\infty}, \\
& \leq \frac{1}{n}\left\|\tilde{\Phi}^{T}(\tilde{E}-\tilde{\Phi} \widehat{S})\right\|_{\infty}+\frac{1}{n}\left\|\tilde{\Phi}^{T} \tilde{\epsilon}\right\|_{\infty} .
\end{aligned}
$$

As the Dantzig constraint:

$$
\left\|\frac{1}{n} \tilde{\Phi}^{T}(\tilde{E}-\tilde{\Phi} \widehat{S})\right\|_{\infty} \leq \tilde{r}
$$

holds, see [19], we have:

$$
\|\Psi \Delta\|_{\infty} \leq \frac{3 \tilde{r}}{2}
$$

\section{Step 4: Control of $\|\Delta\|_{1}$.}

Step 4a. Majoration of $\Delta^{T} \Psi \Delta$. We have, on the event $\mathcal{A} \cap \mathcal{B}$ :

$$
\begin{aligned}
\left|\Delta^{T} \Psi \Delta\right| & \leq\|\Psi \Delta\|_{\infty}\|\Delta\|_{1} \\
& \leq \frac{3 \tilde{r}}{2}\left(\left\|\Delta_{J}\right\|_{1}+\left\|\Delta_{J^{c}}\right\|_{1}\right),
\end{aligned}
$$

by introducing the $\Delta_{J}$ and $\Delta_{J^{c}}$ vectors defined by:

$$
\left(\Delta_{J}\right)_{i}=\left\{\begin{array}{l}
\Delta_{i} \text { if } S_{i} \neq 0 \\
0 \text { else }
\end{array} \quad\left(\Delta_{J^{c}}\right)_{i}=\left\{\begin{array}{l}
0 \text { if } S_{i} \neq 0 \\
\Delta_{i} \text { else }
\end{array}\right.\right.
$$

We recall that $\left\|\Delta_{J^{c}}\right\|_{1} \leq 3\left\|\Delta_{J}\right\|_{1}$ (see [19], Lem. 1, (9)). Hence, on $\mathcal{A} \cap \mathcal{B}$,

$$
\left|\Delta^{T} \Psi \Delta\right| \leq 6 \tilde{r}\left\|\Delta_{J}\right\|_{1} .
$$

Step 4b. Minoration of $\Delta^{T} \Psi \Delta$. Let's introduce the circulant matrix $M$ :

$$
M=\left(\begin{array}{cccc}
1 & \mu & \cdots & \mu \\
\mu & 1 & \ddots & \vdots \\
\vdots & \ddots & \ddots & \mu \\
\mu & \cdots & \mu & 1
\end{array}\right)
$$


whose smallest eigenvalue is $1-\mu$ (see [14]). Hence:

$$
\begin{aligned}
\Delta^{T} \Psi \Delta & =\Delta^{T} M \Delta+\Delta^{T}(\Psi-M) \Delta \\
& \geq(1-\mu)\|\Delta\|_{2}^{2}-\left|\Delta^{T}(\Psi-M) \Delta\right| \\
& \geq(1-\mu)\left\|\Delta_{J}\right\|_{2}^{2}-\left|\Delta^{T}(\Psi-M) \Delta\right| \\
& \geq \frac{1-\mu}{s}\left\|\Delta_{J}\right\|_{1}^{2}-\left|\Delta^{T}(\Psi-M) \Delta\right|,
\end{aligned}
$$

since $\Delta_{J}$ has $s$ nonzero components. We have:

$$
\left|\Delta^{T}(\Psi-M) \Delta\right| \leq\|\Delta\|_{1}\|(\Psi-M) \Delta\|_{\infty} \leq 4\left\|\Delta_{J}\right\|_{1}\|(\Psi-M) \Delta\|_{\infty} .
$$

Now define the event:

$$
\mathcal{C}=\left\{\max _{j \neq i}\left|\Psi_{i j}-\mu\right| \geq \delta\right\} .
$$

It is clear that, on $\mathcal{B} \cap \mathcal{C}$, all entries of $\Psi-M$ are absolutely bounded by $\delta$. Hence, on $\mathcal{B} \cap \mathcal{C}$,

$$
\|(\Psi-M) \Delta\|_{\infty} \leq \delta\|\Delta\|_{1} \leq 4 \delta\left\|\Delta_{J}\right\|_{1}
$$

and, by (A.4):

$$
\left|\Delta^{T}(\Psi-M) \Delta\right| \leq 16 \delta\left\|\Delta_{J}\right\|_{1}^{2}
$$

which gives:

$$
\Delta^{T} \Psi \Delta \geq\left(\frac{1-\mu}{s}-16 \delta\right)\left\|\Delta_{J}\right\|_{1}^{2}
$$

Step 4c. Majoration of $\|\Delta\|_{1}$. By using (A.3) and (A.5), we get that on $\mathcal{A} \cap \mathcal{B} \cap \mathcal{C}$ :

$$
\left\|\Delta_{J}\right\|_{1} \leq \frac{6 \tilde{r}}{\frac{1-\mu}{s}-16 \delta},
$$

hence:

$$
\|\Delta\|_{1} \leq \frac{24 \tilde{r}}{\frac{1-\mu}{s}-16 \delta} .
$$

\section{Step 5: Majoration of $\|\Delta\|_{\infty}$.}

On $\mathcal{A} \cap \mathcal{B} \cap \mathcal{C}$, we have:

$$
\begin{aligned}
\|\Delta\|_{\infty} & \leq\|\Psi \Delta\|_{\infty}+\|\Psi \Delta-\Delta\|_{\infty} \\
& \leq \frac{3 \tilde{r}}{2}+\|(\Psi-\mathrm{Id}) \Delta\|_{\infty} \quad \text { by using (A.2) } \\
& \leq \frac{3 \tilde{r}}{2}+(\mu+\delta)\|\Delta\|_{1} \quad \text { since each entry in } \Psi-\text { Id is less than } \mu+\delta \\
& \leq\left(\frac{3}{2}+\frac{24(\mu+\delta)}{\frac{1-\mu}{s}-16 \delta}\right) \tilde{r} \quad \text { by using (A.6). }
\end{aligned}
$$

To finish, it is easy to see, using Step 2 , that $\mathbb{P}(\mathcal{A} \cap \mathcal{B} \cap \mathcal{C}) \geq 1-\alpha$. 


\section{A.2. Proof of Theorem 2.8}

We rely on the result of [19]. Observe that:

$$
\Psi=\frac{1}{n} \Phi^{T} \Phi
$$

We have for all $j=1, \ldots, p, j \neq i$ :

$$
\mathbb{E}\left(\Psi_{i j}\right)=\frac{1}{n} \sum_{k=1}^{n} \mathbb{E}\left(\tilde{\Phi}_{k i} \tilde{\Phi}_{k j}\right)=0 .
$$

Hence, for any $\delta>0$, Hoeffding's inequality and union bound give:

$$
\mathbb{P}\left(\max _{j \neq i}\left|\Psi_{i j}\right| \geq \delta\right) \leq \exp \left(-n \frac{\delta^{2}}{2}+2 \ln p\right) .
$$

We also notice that $\Psi_{i i}=1$ for all $i$. Hence, Assumptions 1 and 2 of (Thm. 1 in [19]) are satisfied with probability as described in the statement of the theorem.

\section{ApPendix B. Support RECOVERY USING THRESHOLDED-LASSO}

\section{B.1. Standard results using UDP}

Lemma B.1 (Lem. A.2 in [8]). Let $r>r_{0}>0$ and $\hat{S}$ a solution to (2.7) with regularizing parameter $r$. Set $\Delta=\hat{S}-S$. On the event $\left\{(1 / n)\left\|\Phi^{\top} \epsilon\right\|_{\infty} \leq r_{0}\right\}$, it holds that for all $T \subseteq\{1, \ldots, p\}$ such that $|T| \leq s$,

$$
\frac{1}{2 r}\left[\frac{1}{2 n}\|\Phi \Delta\|_{2}^{2}+\left(r-r_{0}\right)\|\Delta\|_{1}\right] \leq\left\|\Delta_{T}\right\|_{1}+\left\|S_{T^{c}}\right\|_{1} .
$$

Proof. By optimality in (2.7), we get:

$$
\frac{1}{2 n}\|E-\Phi \hat{S}\|_{2}^{2}+r\|\hat{S}\|_{1} \leq \frac{1}{2 n}\|\epsilon\|_{2}^{2}+r\|S\|_{1} .
$$

It yields,

$$
\frac{1}{2 n}\|\Phi \Delta\|_{2}^{2}-\frac{1}{n}\left\langle\Phi^{\top} \epsilon, \Delta\right\rangle+r\|\hat{S}\|_{1} \leq r\|S\|_{1} .
$$

Let $T \subseteq\{1, \ldots, p\}$ such that $|T| \leq s$. We assume that $(1 / n)\left\|\Phi^{\top} \epsilon\right\|_{\infty} \leq r_{0}$. Invoking Hölder's inequality, we have:

$$
\frac{1}{2 n}\|\Phi \Delta\|_{2}^{2}+r\left\|\hat{S}_{J^{c}}\right\|_{1} \leq r\left(\left\|S_{J}\right\|_{1}-\left\|\hat{S}_{J}\right\|_{1}\right)+r\left\|S_{J^{c}}\right\|_{1}+r_{0}\|\Delta\|_{1} .
$$

Adding $r\left\|S_{J^{c}}\right\|_{1}$ on both sides, observe that:

$$
\frac{1}{2 n}\|\Phi \Delta\|_{2}^{2}+\left(r-r_{0}\right)\left\|\Delta_{J^{c}}\right\|_{1} \leq\left(r+r_{0}\right)\left\|\Delta_{J}\right\|_{1}+2 r\left\|S_{J^{c}}\right\|_{1} .
$$

Adding $\left(r-r_{0}\right)\left\|\Delta_{J}\right\|_{1}$ on both sides, we conclude the proof.

Lemma B.2 (Thm. 2.1 in [8]). Assume that for all $\gamma \in \mathbb{R}^{p}$, for all $T \subseteq\{1, \ldots, p\}$ such that $|T| \leq s$,

$$
\left\|\gamma_{T}\right\|_{1} \leq \rho \sqrt{s}\|\Phi \gamma\|_{2}+\kappa\|\gamma\|_{1} .
$$

where $\rho>0$ and $1 / 2>\kappa>0$. Moreover, assume that the regularizing parameter $r$ of the convex program (2.7) enjoys $r>r_{0} /(1-2 \kappa)$. Then, on the event $\left\{(1 / n)\left\|\Phi^{\top} \epsilon\right\|_{\infty} \leq r_{0}\right\}$, any solution $\hat{S}$ to (2.7) satisfies:

$$
\|\hat{S}-S\|_{1} \leq \frac{2 r n \rho^{2} s}{1-\left(r_{0} / r\right)-2 \kappa} .
$$


Proof. Assume that $(1 / n)\left\|\Phi^{\top} \epsilon\right\|_{\infty} \leq r_{0}$. Using (3.1) and (B.1) with $J=T$, the support of $S$, we get:

$$
\frac{1}{2 r}\left[\frac{1}{2 n}\|\Phi \Delta\|_{2}^{2}+\left(r-r_{0}\right)\|\Delta\|_{1}\right] \leq \rho \sqrt{s}\|\Phi \Delta\|_{2}+\kappa\|\Delta\|_{1},
$$

where $\Delta=\hat{S}-S$. It yields,

$$
\left[\frac{1}{2}\left(1-\frac{r_{0}}{r}\right)-\kappa\right]\|\Delta\|_{1} \leq-\frac{1}{4 r n}\|\Phi \Delta\|_{2}^{2}+\rho \sqrt{s}\|\Phi \Delta\|_{2} \leq r n \rho^{2} s
$$

using the fact that the polynomial $x \mapsto-1 /(4 r n) x^{2}+\rho \sqrt{s} x$ is not greater than $r n \rho^{2} s$.

\section{B.2. Rademacher designs}

The result and the proof given on the 13th page rely on the following lemmas.

Lemma B.3 (Rademacher designs satisfy RIP). There exists universal constants $c_{1}, c_{2}, c_{3}$ such that the following holds. Let $\delta \in(0,1)$ and $p, n, s^{\prime}>0$ such that:

$$
s^{\prime}=\left\lfloor\frac{c_{1} \delta^{2} n}{\log \left(c_{2} p /\left(\delta^{2} n\right)\right)}\right\rfloor
$$

then, with probability at least $1-2 \exp \left(-c_{3} n\right)$, a matrix $\Phi \in\{ \pm 1\}^{n \times p}$ drawn according to the Rademacher's model (2.12) enjoy the RIP property, namely for all $\gamma \in \mathbb{R}^{p}$ such that $\|\gamma\|_{0} \leq s^{\prime}$,

$$
n(1-\delta)^{2}\|\gamma\|_{2}^{2} \leq\|\Phi \gamma\|_{2}^{2} \leq n(1+\delta)^{2}\|\gamma\|_{2}^{2} .
$$

Proof. Numerous authors have proved this result, see Example 2.6.3 and Theorem 2.6.5 in [7] for instance.

Lemma B.4 (Rademacher designs satisfy UDP). There exists universal constants $c_{1}, c_{2}, c_{3}$ such that the following holds. Let $\delta \in(0, \sqrt{2}-1)$ and $s>0$ such that:

$$
5 s \leq s^{\prime}:=\left\lfloor\frac{c_{1} \delta^{2} n}{\log \left(c_{2} p /\left(\delta^{2} n\right)\right)}\right\rfloor,
$$

then, with probability at least $1-2 \exp \left(-c_{3} n\right)$, a matrix $\Phi \in\{ \pm 1\}^{n \times p}$ drawn according to the Rademacher model (2.12) enjoy for all $\gamma \in \mathbb{R}^{p}$ and for all $T \subseteq\{1, \ldots, p\}$ such that $|T| \leq s$,

$$
\left\|\gamma_{T}\right\|_{1} \leq \rho \sqrt{s}\|\Phi \gamma\|_{2}+\kappa\|\gamma\|_{1} .
$$

where:

- $1 / 2>\kappa>\left(1+2((1-\delta) /(1+\delta))^{\frac{1}{2}}\right)^{-1}$,

- $\sqrt{n} \rho=\left(\sqrt{1-\delta}+\left(\kappa_{0}-1\right) /\left(2 \kappa_{0}\right) \sqrt{1+\delta}\right)^{-1}$.

Proof. The proof follows from Lemma B.3 and Proposition 3.1 in [8].

Lemma B.5. Let $c, C_{1}>0$ and $p, n, s>0$ such that $n \geq C_{1} s \log p$ and $s \geq 3(2+c) / C_{1}$. Then, with probability greater than $1-2 p^{-c}$, it holds for all $k \neq l \in\{1, \ldots, p\}$,

$$
\frac{1}{n}\left|\sum_{i=1}^{n} \Phi_{i, k} \Phi_{i, l}\right| \leq\left[\frac{(2+c) 8}{3 C_{1}}\right]^{1 / 2} \frac{1}{\sqrt{s}} .
$$


Proof. Let $k \neq l \in\{1, \ldots, p\}$. Set $X_{i}=\Phi_{i, k} \Phi_{i, l}$ and observe that $X_{i}$ are independent Rademacher random variables. From Bernstein's inequality, it holds for all $0<t<1$,

$$
\mathbb{P}\left(\left|\frac{1}{n} \sum_{i=1}^{n} X_{i}\right| \geq t\right) \leq 2 \exp \left(-\frac{3 n}{8} t^{2}\right) .
$$

Set $t=\left((2+c) 8 /\left(3 s C_{1}\right)\right)^{1 / 2}$ and observe $\#\{k \neq l\} \leq \exp (2 \log p)$.

Acknowledgements. We would like to thank the anonymous referees, whose careful reading and comments have helped to greatly improve this paper.

\section{REFERENCES}

[1] P.J. Bickel, Y. Ritov and A.B. Tsybakov, Simultaneous analysis of Lasso and Dantzig selector. Ann. Stat. 37 (2009) $1705-1732$.

[2] P.J. Bickel, Y. Ritov and A.B. Tsybakov, Simultaneous analysis of lasso and Dantzig selector. Ann. Stat. 37 (2009) $1705-1732$.

[3] P.L. Bühlmann and S.A. van de Geer, Statistics for High-Dimensional Data. Springer (2011).

[4] E.J. Candès and Y. Plan, Near-ideal model selection by L1 minimization. Ann. Stat. 37 (2009) 2145-2177.

[5] E.J. Candes and T. Tao, The Dantzig selector: statistical estimation when $p$ is much larger than $n$. Ann. Stat. 35 (2007) 2313-2351.

[6] E.J. Candes, J.K. Romberg and T. Tao, Stable signal recovery from incomplete and inaccurate measurements. Commun. Pure Appl. Math. 59 (2006) 1207-1223.

[7] D. Chafal, O. Guédon, G. Lecué and A. Pajor, Interactions Between Compressed Sensing, Random Matrices, and High Dimensional Geometry. Panoramas et Synthèses. SMF (2012).

[8] Y. de Castro, A remark on the lasso and the Dantzig selector. Stat. Probab. Lett. (2012).

[9] D.L. Donoho, M. Elad and V.N. Temlyakov, Stable recovery of sparse overcomplete representations in the presence of noise. Inf. Theory IEEE Trans. 52 (2006) 6-18.

[10] B. Efron, T. Hastie, I. Johnstone and R.J. Tibshirani, Least angle regression. Ann. Stat. 32 (2004) $407-499$.

[11] Jianqing Fan, Yang Feng and Rui Song, Nonparametric independence screening in sparse ultra-high-dimensional additive models. J. Am. Stat. Assoc. 106 (2011).

[12] J.-J. Fuchs, On sparse representations in arbitrary redundant bases. Inf. Theory IEEE Trans. 50 (2004) 1341-1344.

[13] F. Gamboa, A. Janon, T. Klein, A. Lagnoux-Renaudie and C. Prieur, Statistical inference for Sobol pick freeze Monte Carlo method. Preprint arXiv: 1303.6447 (2013).

[14] R. Gray, Toeplitz and Circulant Matrices: A Review. Now Publishers Inc. (2006).

[15] W. Hoeffding, Probability Inequalities for Sums of Bounded Random Variables. J. Am. Statist. Assoc. 58 (1963) 13-30.

[16] A. Janon, T. Klein, A. Lagnoux, M. Nodet and C. Prieur, Asymptotic normality and efficiency of two Sobol index estimators. Preprint available at http://hal.inria.fr/hal-00665048/en (2012).

[17] A. Juditsky and A. Nemirovski, Accuracy Guarantees for-Recovery. Inf. Theory IEEE Trans. 57 (2011) 7818-7839.

[18] R. Liu and A.B. Owen, Estimating Mean Dimensionality. Department of Statistics, Stanford University (2003).

[19] K. Lounici, Sup-norm convergence rate and sign concentration property of Lasso and Dantzig estimators. Electron. J. Stat. 2 (2008) 90-102.

[20] H. Monod, C. Naud and D. Makowski, Uncertainty and sensitivity analysis for crop models. In Chap. 4. Working with Dynamic Crop Models: Evaluation, Analysis, Parameterization, and Applications. Edited by D. Wallach, D. Makowski and J.W. Jones. Elsevier (2006) 55-99.

[21] M.D. Morris, Factorial sampling plans for preliminary computational experiments. Technometrics 33 (1991) $161-174$.

[22] A. Saltelli, M. Ratto, T. Andres, F. Campolongo, J. Cariboni, D. Gatelli, M. Saisana and S. Tarantola, Global Sensitivity Analysis: The Primer. Wiley Online Library (2008).

[23] I.M. Sobol, Sensitivity estimates for nonlinear mathematical models. Math. Model. Comput. Experiment 1 (1993) $407-414$.

[24] I.M. Sobol, Global sensitivity indices for nonlinear mathematical models and their Monte Carlo estimates. Math. Comput. Simul. 55 (2001) 271-280.

[25] S. Tarantola et al., Estimating the approximation error when fixing unessential factors in global sensitivity analysis. Reliab. Eng. Syst. Safety 92 (2007) 957-960. 
[26] R.J. Tibshirani, Regression shrinkage and selection via the lasso. J. R. Stat. Soc. Ser. B. Methodological (1996) $267-288$.

[27] J.-Y. Tissot and C. Prieur, Bias correction for the estimation of sensitivity indices based on random balance designs. Reliab. Eng. Syst. Safety 107 (2012) 205-213.

[28] J.-Y. Tissot and C. Prieur, Estimating Sobol'Indices Combining Monte Carlo Estimators and Latin Hypercube Sampling (2012).

[29] J.A. Tropp, Just relax: Convex programming methods for identifying sparse signals in noise. Inf. Theory IEEE Trans. 52 (2006) 1030-1051.

[30] S.A. van de Geer and P. Bühlmann, On the conditions used to prove oracle results for the Lasso. Electron. J. Stat. 3 (2009) 1360-1392.

[31] L. Welch, Lower bounds on the maximum cross correlation of signals (Corresp.). Inf. Theory, IEEE Trans. 20 (1974) $397-399$.

[32] P. Zhao and B. Yu, On model selection consistency of Lasso. J. Mach. Learn. Res. 7 (2006) 2541-2563. 